\title{
A high-efficiency hospital emergency-response mode is key to successful treatment of COVID-19 patients in Zhuhai
}

\section{Jin Huang}

Fifth Affiliated Hospital of Sun Yat-sen University

\section{Zhonghe Li}

Fifth Affiliated Hospital of Sun Yat-sen University

\section{Xiujuan Qu}

Fifth Affiliated Hospital of Sun Yat-sen University

\section{Xiaobin Zheng}

Fifth Affiliated Hospital of Sun Yat-sen University

\section{Changli Tu}

Fifth Affiliated Hospital of Sun Yat-sen University

\section{Honglei Shi}

Fifth Affiliated Hospital of Sun Yat-sen University

\section{Meizhu Chen}

Fifth Affiliated Hospital of Sun Yat-sen University

\section{Cuiyan Tan}

Fifth Affiliated Hospital of Sun Yat-sen University

Jing Liu ( $\square$ liujing25@sysu.edu.cn )

Fifth Affiliated Hospital of Sun Yat-sen University

\section{Hong Shan}

Fifth Affiliated Hospital of Sun Yat-sen University

\section{Research}

Keywords: hospital emergency-response mode, NCP department, treatment, COVID-19, high-efficiency

Posted Date: April 2nd, 2020

DOI: https://doi.org/10.21203/rs.3.rs-20125/v1

License: (c) (1) This work is licensed under a Creative Commons Attribution 4.0 International License. Read Full License 


\section{Abstract}

Background: In December 2019, coronavirus disease 2019 (COVID-19) emerged in Wuhan and has since rapidly spread throughout China. The mortality rates of novel coronavirus pneumonia (NCP) in severe and critical cases are very high. In this public-health emergency, a high-efficiency administrative emergency-response mode in designated hospitals is needed.

Method: As an affiliated hospital of Sun Yat-sen University, ours, the Fifth Affiliated Hospital, is the only one designated for the diagnosis and treatment of COVID-19 in Zhuhai, a mid-sized city. The NCP department, for which the president of the hospital is also the direct administrative lead, was established at an early stage of the epidemic at our hospital. This department includes core members of the pulmonary and critical-care medicine (PCCM) specialist and multidisciplinary team. Rather than adhering to national guidelines on NCP, we have focused on individualized treatment, timely adjustment thereof and management strategies in working with COVID-19 patients based on the professional opinions of a professor of respiratory medicine and an expert group.

Results: (1) High working efficiency: As of March 2, 2020, we have completed 2974 citywide consultations and treatment of 366 inpatients, including 101 who were diagnosed with COVID-19. (2) Excellent therapeutic effect: Of the 101 patients hospitalized with confirmed COVID-19, only 1 has died, and the rest were all cured and discharged. No secondary hospital infection, pipeline infection or pressure sores were found in any patient. (3) Finding and confirming person-to-person transmission characteristic of COVID-19 prior to the official press conference: Strengthened protection is key to zero infection among the healthcare providers and medical faculty, as well as to a lower rate of second-generation infectious patients. (4) Timely adjustment of management and treatment strategy prior to guideline updates: The first evidence of digestive-tract involvement in COVID-19 has been found, and the earliest clinical trial of chloroquine in the treatment of the disease was carried out at our hospital.

Conclusions: At our hospital, establishment of an NCP department, which is directly administered by the hospital president and specialized operation guided by a professor of respiratory medicine, has been key to our success in managing and treating COVID-19 patients. Our hospital's emergency-response mode could provide a reference for other hospitals and cities in this epidemic situation.

\section{Contributions To Literature}

- We can comprehensively recognize the shortcomings and deficiencies of traditional emergencyresponse system in the capacity to recognize new infectious diseases.

- The key to our success in managing and treating COVID-19 patients is to establish NCP department with the professional guidance from the PCCM department, and multidisciplinary participation. Our high-efficiency hospital emergency-response mode can provide reference for other hospitals and regions. 
- In the treatment process, on the basis of the national guidelines, the treatment plan should be adjusted in time according to the opinions of the professor of respiratory medicine and an expert group under the direct authorization of the president, and therapeutic effect will be better with the individualized treatment.

\section{Introduction:}

In December 2019, COVID-19 emerged in Wuhan and has since rapidly spread throughout China and the world. As of March 26, 2020, there were 81,961 confirmed patients and 3,293 deaths in 34 provinces in China. Outside of China, there were 380,723 confirmed patients and 17,541 deaths in 197 countries $^{1}$. In different regions and different hospitals, there are different management models for infectious respiratory diseases, meaning that the treatment effects are different too ${ }^{2}$. At the beginning of this new epidemic, the absence or insufficiency of hospital emergency-response plans caused a rapid spread of COVID-19 and high mortality rates in severely ill COVID-19 patients, which has greatly increased the subsequent costs of fighting the epidemic nationwide ${ }^{3}$. Therefore, some effective hospital management models need to be shared, summarized and referenced in order to stop the epidemic as soon as possible and reduce mortality.

\section{Method:}

Zhuhai, a mid-sized city in Guangdong Province, has 72 public medical and health institutions, including four tertiary hospitals. The authors' hospital, the Fifth Affiliated Hospital of Sun Yat-sen University, is the only one designated for the diagnosis and treatment of COVID-19 patients in Zhuhai; it is responsible for consultation on and centralized treatment of these patients. When the first patient was admitted on January 17,2020 , our hospital activated an emergency plan for infectious diseases. It was still employing the traditional emergency-response mode: patients were admitted to the Department of Infectious Diseases, receiving consultation by the PCCM department and instruction from the administrative department (Fig. 1). However, after 4 days, the treatment effect for COVID-19 patients was assessed as poor and the hospital's work efficiency in relation to this disease as low.

The president of the hospital quickly made a new decision. Under his direct administrative leadership and management and with the professional guidance of the PCCM department, the NCP department was established. The Department of Infectious Diseases was given the more peripheral role of handling infection control, severely and critically ill patients received multidisciplinary consultation, the hospital's professor of PCCM decided on a finalized treatment strategy, and the entire hospital was mobilized to serve the NCP department first and foremost and to fight the COVID-19 crisis (Fig. 2). Rather than adhering to the national guidelines on NCP, we focused on individualized treatment, timely adjustment thereof and management strategies in working with COVID-19 patients, based on the professional opinions of a professor of respiratory diseases and an expert group. 


\section{Results:}

\section{High working efficiency:}

As of March 2, 2020, we have completed 2974 citywide consultations and treated 366 inpatients, including 101 who were diagnosed with COVID-19. The average age of these 101 patients is $45 \pm 18.01$ years, 47 are males and 87 of cases are from Hubei Province. When the NCP department was first established, five treatment areas (with a total of 80 doctors and 150 nurses) were set up in 3 days, each able to accommodate 17 patients in isolation; this ensures that all of the suspected patients in Zhuhai can be isolated and diagnosed in time. Subsequently, a total of 10 treatment areas (with a total of 162 doctors and 322 nurses) were successively established within 1 week, including one respiratory intensivecare unit (RICU; for critically ill patients), two treatment areas for severely ill patients, three for non-severe COVID-19 patients, two for suspected cases, one transitional area after performance of nucleic-acid removal testing, and one isolation area before patient discharge.

\section{Excellent therapeutic effect:}

Of the 101 hospitalized patients with confirmed COVID-19, 26 severely ill patients had a ratio of arterial oxygen partial pressure to fractional inspired oxygen ( $\mathrm{P} / \mathrm{F}$ ratio) $<300 \mathrm{mmHg}$, while 10 critically ill patients had a $\mathrm{P} / \mathrm{F}$ ratio $<150 \mathrm{mmHg}$ accompanied by elevated lactate dehydrogenase (LDH) levels. Despite the high rate of severe/critical cases $(25.74 \%$ ) at our hospital, all but one patient were cured and discharged (Fig. 3). The remaining patient died after having been given invasive mechanical ventilation too early due to the NCP department not yet having been established.

Our knowledge and understanding of COVID-19 are continually being supplemented and updated. In the NCP department, the professor from the Department of Respiratory Medicine is directly responsible for the treatment plan, individualizing treatment according to patients' specific conditions rather than sticking to national guidelines; this has been the key to our success in treating 100 of the 101 total patients. The effective implementation of the treatment plan has benefited from the direct leadership and instruction of the hospital president. Moreover, due to strict discharge standards, the relapse rate of patients after discharge is only $0.97 \%$. Moreover, no secondary hospital infection, pipeline infection or pressure sores have been found in severely ill patients (Fig. 3), because 20 doctors, 33 nurses, and adequate protective materials and equipment have been supplied to the RICU.

3. Finding and confirming person-to-person transmission characteristic of COVID-19 prior to official press conference:

We have discovered, confirmed and reported such transmissions before holding an official press conference. Subsequently, the local People's Health Commission and administrative departments in Zhuhai were promptly informed of these transmissions, and a prompt response plan and stronger protection against the virus were established. The family and close contacts of confirmed COVID-19 patients were isolated quickly, so that there were only 16 second-generation infectious patients (local to 
Zhuhai), comprising only $15.53 \%$ of all 101 patients. Strengthened anti-viral protection has also been key to the zero rate of infection among our healthcare providers and medical faculty (Fig. 3). In the NCP department, 300 sets of protective N95 masks and protective suits have been used per day. In addition, a total of 1500 sets of protective gear, including nine positive-pressure electric supplied-air respirators, have been used in the RICU.

4. Timely adjustment of management and treatment strategy prior to guideline updates: The first evidence of digestive-tract involvement in COVID-19 was found at our hospital ${ }^{4}$, which improved the discharge process for COVID-19 patients. Discharge standards based on national guidelines consider only elimination of 2019 novel coronavirus (2019-nCoV) from the respiratory tract, but we found that 2019-nCoV remained longer in the digestive tract than in the respiratory tract in some patients.

Therefore, we do not permit discharge until 2019-nCoV has been eliminated from both stool and the respiratory tract, which has greatly lowered the rate of relapse. In addition, the earliest clinical trial of chloroquine in the treatment of COVID-19 was carried out in our hospital, which was helpful for the inclusion of chloroquine in the updated guidelines.

\section{Discussion:}

Different countries employ different emergency systems to deal with certain public-health crises. For example, the Hospital Emergency Incident Command System (HEICS) has emerged as a popular model of hospital emergency response in the United States and other some countries. Since the inception of the HEICS in 1991, several events have transformed the requirements of hospital emergency management, including the 1995 Tokyo Subway sarin attack and the 2003 severe acute respiratory syndrome (SARS) outbreaks in eastern Asia and Toronto, Canada. HEICS, which has certain disadvantages, should be continuously improved as additional challenges arise and as hospitals gain more experience with its use $^{5}$. However, in China, most hospitals still lack an emergency management system similar to HEICS. Over the past 20 years, the major infectious respiratory diseases in China have been the SARS outbreaks in 2003, the H1N1 crisis in 2009 and small-scale prevalence of H7N9 in 2013. In terms of epidemic and transmission scale, the COVID-19 crisis resembles SARS, so lessons learned from the SARS epidemic should be especially important in responding to the current emergency ${ }^{6}$. In fact, significant deficiencies in the capacity to recognize new infectious diseases and an imperfect emergency-response system were found in China ${ }^{7}$. Unfortunately, these deficiencies have not been given more attention and improved over time, leading to a potential crisis.

Zhuhai has a permanent-resident population of about 1.89 million, but the proportion of the transient population is higher, making COVID-19 difficult to protect against and control. Our hospital is the only one in Zhuhai designated to fight this disease. There are advantages and disadvantages to dedicating a comprehensive tertiary hospital to the management and treatment of COVID-19 patients. Compared with a dedicated infectious-disease hospital, a comprehensive tertiary hospital has a powerful PCCM department and multidisciplinary consultation and can mobilize more resources. For example, at our hospital, extracorporeal-membrane oxygenation (ECMO) is performed by a group of clinicians with rich 
experience therein, and our surgical department completed thoracic closed-drainage tracheotomy in a COVID-19 patient. However, the disadvantages of too many administrative and related clinical departments involved in the traditional hospital response mode, include departmental conflicts of interest, lack of enforcement, low work efficiency in treatment and management of COVID-19 patients.

Nonetheless, our hospital was able to assess the epidemic situation, quickly regulate hospital strategy and establish the NCP department under the direct leadership and management of the hospital president, along with professional guidance from the faculty of the PCCM department. The Department of Infectious Diseases was transferred to the periphery by being put in charge of infection control instead of NCP treatment. Based on our respiratory specialist's rich experience in treating viral pneumonia, our COVID-19 patients have received good treatment, particularly the 26 who were severely or critically ill. In 1 month, six versions of the national guidelines for NCP have been published. Rather than sticking to these guidelines, we have implemented individualized treatment based on the opinions of the professor of respiratory medicine and an expert group under the direct authorization of the president. Therefore, after we adjusted management mode and treatment strategy, the overall treatment effect significantly improved, and all patients were successfully cured and discharged.

Meanwhile, we achieved an infection rate of zero among our healthcare providers and medical faculty due to early discovery of person-to-person transmission of COVID-19, sufficient supplies of protective equipment and materials, and governmental financial support. In addition, under the direct leadership of the president, the scientific-research team in the NCP department has responded quickly to problems found in clinical practice. The first evidence of digestive-tract involvement in COVID-19 was found via gastroscopy and colonoscopy at our hospital, which improved the discharge process and lowered the relapse rate in COVID-19 patients. We were also responsible for the earliest application of chloroquine as an anti-viral drug in COVID-19 due to the direct instruction of the hospital president.

\section{Conclusions】}

In our hospital, establishment of the NCP department, which is directly administered by the hospital's president and guided by a professor of respiratory medicine, has been the key to our success in managing and treating COVID-19 patients. In the treatment process, on the basis of the national guidelines, the treatment plan was adjusted in time according to the opinions of the professor of respiratory medicine and an expert group under the direct authorization of the president, and therapeutic effect was better with the individualized treatment. Our hospital's emergency-response mode could provide a reference for other hospitals and cities in this epidemic situation.

\section{Abbreviations}

COVID-19

coronavirus disease 2019

NCP 
novel coronavirus pneumonia

PCCM

pulmonary and critical-care medicine

RICU

respiratory intensive-care unit

$\mathrm{P} / \mathrm{F}$ ratio

arterial oxygen partial pressure to fractional inspired oxygen

LDH

lactate dehydrogenase

HEICS

the Hospital Emergency Incident Command System

SARS

severe acute respiratory syndrome

ECMO

extracorporeal-membrane oxygenation

\section{Declarations}

\section{Ethics approval and consent to participate}

Not applicable.

\section{Consent for publication}

Not applicable.

\section{Availability of data and material}

All data generated or analyzed during this study are included in this published article.

\section{Competing interests}

The authors declare that they have no competing interests.

\section{Funding}

The authors have not received any funding for this study.

\section{Authors' contributions}

$\mathrm{H}$ S and $\mathrm{J} L$ conceived and designed this study and gave final approval of the version to be published; $\mathrm{H}$ $\mathrm{S}$ and $\mathrm{J} \mathrm{L}$ were both correspondents; $\mathrm{J} \mathrm{H}$ and $\mathrm{ZH} \mathrm{L}$ performed this study and wrote the manuscript, contributing equally to this work as first authors; XJ Q, XB Z and CL T collected and analyzed the data; HL S, MZ C and CY T wrote this paper. All of the authors read and approved the final manuscript. 


\section{*Acknowledgments}

The authors do not have any acknowledgments to make.

\section{References}

1. Novel coronavirus (2019-nCoV). Situation report-56. WHO.

https://www.who.int/emergencies/diseases/novel-coronavirus-2019/situation-reports. Mar 26, 2020

2. Souza DB, Dall'Agnol CM. Public health emergency: social representations among managers of a university hospital. Rev Lat Am Enfermagem. 2013 Jul-Aug; 21(4): 998-1004. doi: 10.1590/S010411692013000400023.

3. Lei Ding, Wei Cai, Jianqing Ding, et al. Ponder over the novel coronavirus infection epidemic situation. SCIENCE CHINA Life Sciences, 2020.2.23 online (in Chinese).

4. Fei Xiao, Meiwen Tang, Xiaobin Zheng, et al. Evidence for Gastrointestinal Infection of SARS-CoV-2. Gastroenterology. 2020 Mar 3. DOI: 10.1053/j.gastro.2020.02.055 [Online ahead of print]

5. Arnold JL, Dembry LM, Tsai MC, et al. Recommended Modifications and Applications of the Hospital Emergency Incident Command System for Hospital Emergency Management. Prehosp Disaster Med, 2005 Sep-Oct; 20 (5), 290-300. DOI: 10.1017/s1049023x00002740

6. Cheng VC, Chan JF, To KK, et al. Clinical management and infection control of SARS: lessons learned. Antiviral Res. 2013 Nov; 100(2):407-19. doi: 10.1016/j.

7. Feng Z, Li W, Varma JK. Gaps remain in China's ability to detect emerging infectious diseases despite advances since the onset of SARS and avian flu. Health Aff (Millwood). 2011 Jan; 30(1):127-35. doi: 10.1377/hlthaff.2010.0606.

\section{Figures}




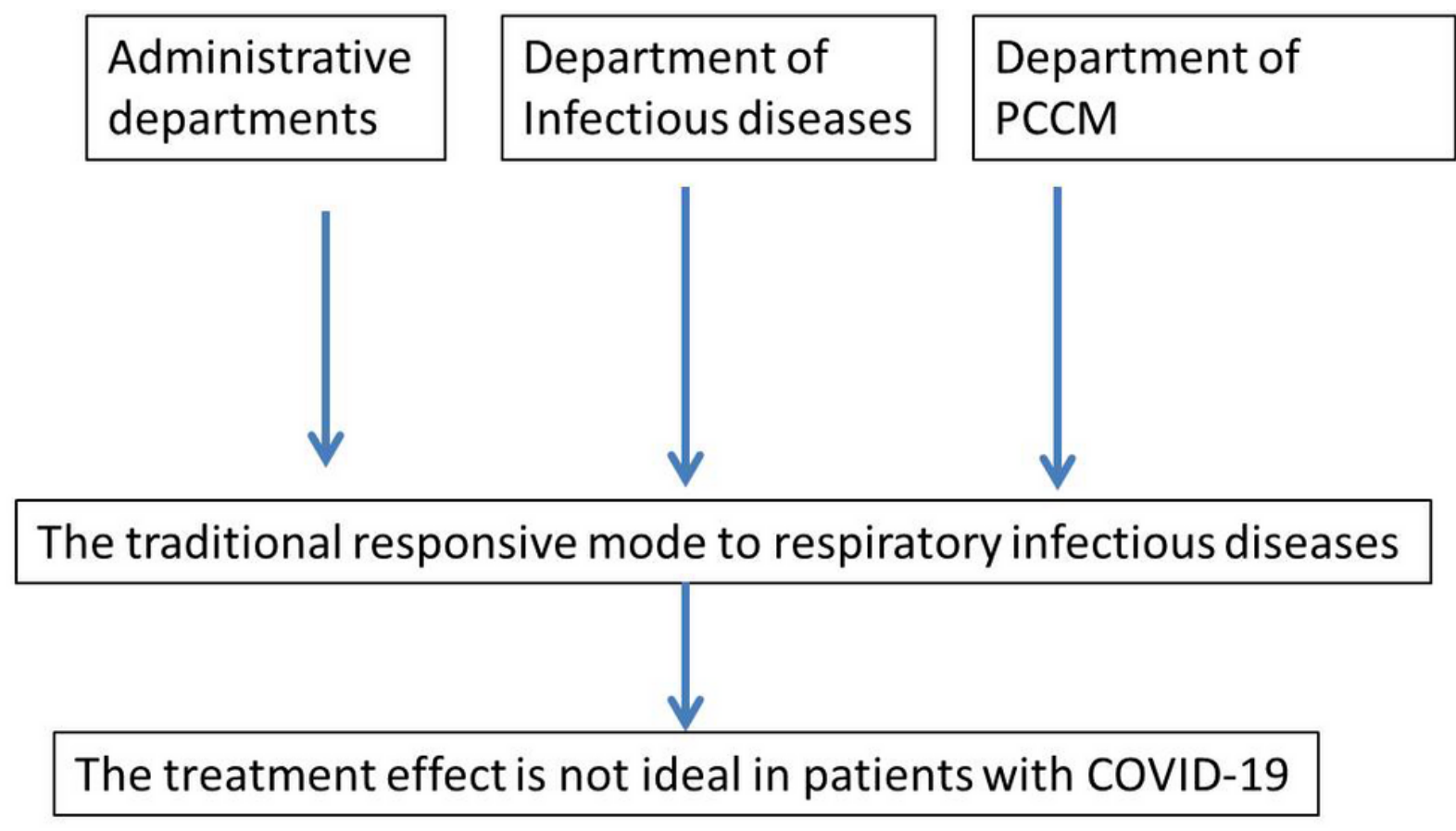

Figure1. Traditional responsive mode to respiratory infectious diseases

PCCM: Pulmonary and Critical Care Medicine ; COVID-19: Coronavirus Disease 2019

Figure 1

Traditional response mode to respiratory infectious diseases. PCCM: pulmonary and critical-care medicine; COVID-19: coronavirus disease 2019. 


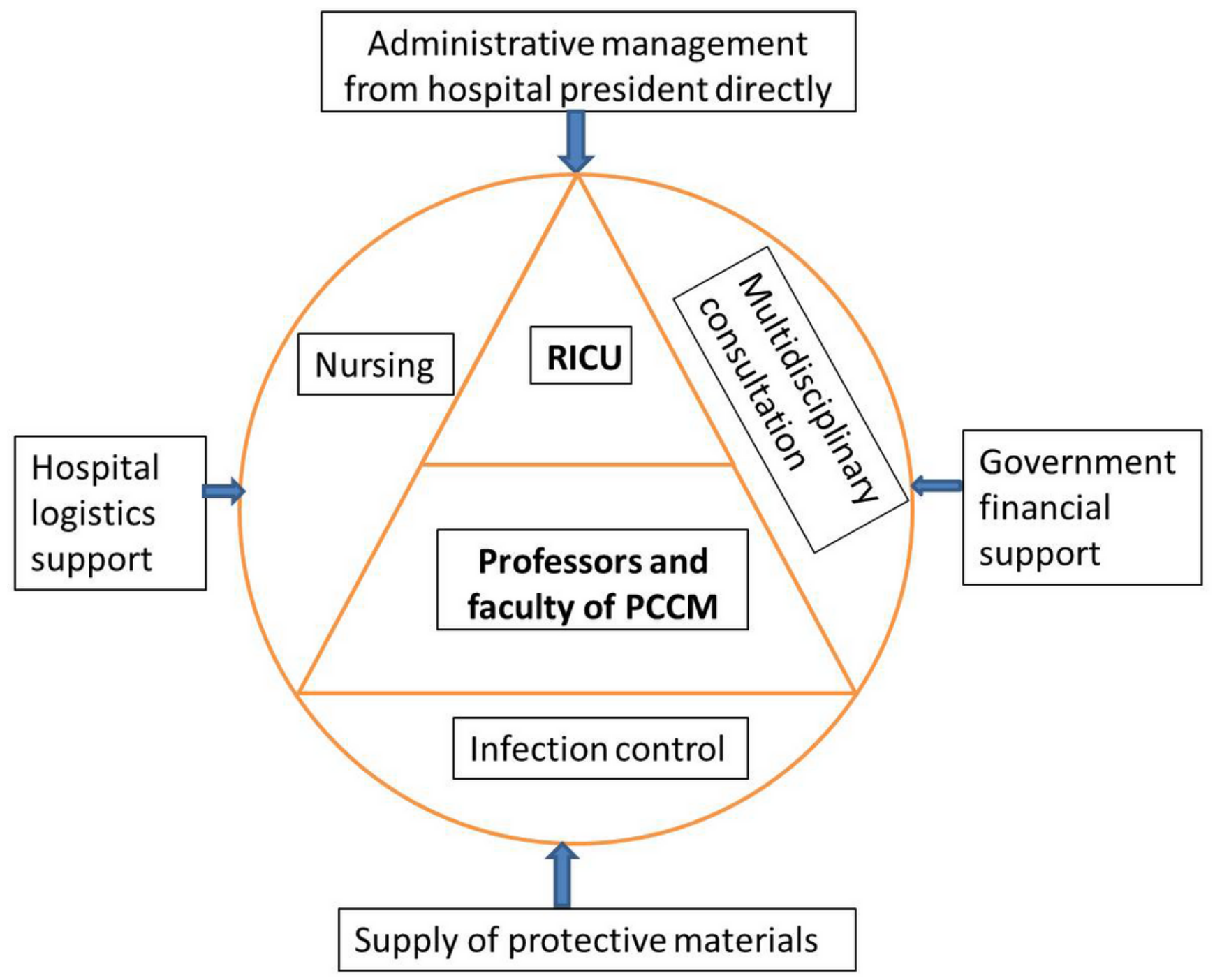

Figure2. A high efficient hospital emergency incident responsive mode:

Establishment of Novel coronavirus pneumonia department

RICU :Respiratory Intensive Care Unit; PCCM: Pulmonary and Critical Care Medicine

Figure 2

A high-efficiency hospital emergency-response mode: establishment of the novel coronavirus pneumonia (NCP) department. RICU: Respiratory Intensive Care Unit; PCCM: Pulmonary and Critical Care Medicine 
Medical faculty

(doctors and nurses)

$n=484$
100 patients were cured and discharged (99\%)

Protective equitment
and material
$(300$ set/d)

No infection in health care staff

No secondary hospital infection, no

Nursing staff in RICU

$n=33$ pipeline infection and no pressure sore were found in severe patients

Figure3. The effect of hospital emergency incident responsive mode in The Fifth Affiliated Hospital of Sun Yat-sen University

Figure 3

Effects of hospital emergency-response mode at The Fifth Affiliated Hospital of Sun Yat-sen University.

\section{Supplementary Files}

This is a list of supplementary files associated with this preprint. Click to download.

- SQUIRE2.0ChecklistforReviewers160315.docx 